\title{
Analysis of the Sulfuric Acid Hydrolysis of Wood Pulp for Cellulose Nanocrystal Production: A Central Composite Design Study
}

Shuping Dong ${ }^{\mathrm{a}, \mathrm{b}}$, Michael J. Bortner ${ }^{\mathrm{b}, \mathrm{c}}$, Maren Roman ${ }^{\mathrm{a}, \mathrm{b}, *}$

aDepartment of Sustainable Biomaterials, Virginia Tech, Blacksburg, VA 24061, USA

${ }^{\mathrm{b}}$ Macromolecules and Interfaces Institute, Virginia Tech, Blacksburg, VA 24061, USA

'Department of Chemical Engineering, Virginia Tech, Blacksburg, VA 24061, USA

*Corresponding Author: Maren Roman, mgrunert@ vt.edu

\begin{abstract}
Cellulose nanocrystals (CNCs) are most commonly prepared by sulfuric acid hydrolysis of a purified cellulose starting material but the effects of hydrolysis conditions on CNC yield and properties are incompletely understood. In this study, we use a rotatable central composite experimental design to elucidate parameter interactions between three design factors, acid concentration $\left(x_{1}\right)$, hydrolysis temperature $\left(x_{2}\right)$, and hydrolysis time $\left(x_{3}\right)$, over a broad range of process conditions and determine their effect on sulfate group density. Parameter ranges are 55$65 \mathrm{wt} . \%$ for $x_{1}, 45-65^{\circ} \mathrm{C}$ for $x_{2}$, and 30-180 min for $x_{3}$. Regression models of the experimental data reveal significant two-factor interactions of $x_{1}$ with each $x_{2}$ and $x_{3}$, whereas $x_{2}$ has no significant two-factor interaction with $x_{3}$. The models predict a maximum yield of $66-69 \%$ at optimum process conditions of 57-58 wt. $\%\left(x_{1}\right), 64-67{ }^{\circ} \mathrm{C}\left(x_{2}\right)$, and 134-156 min $\left(x_{3}\right)$. At these conditions, the sulfate group density is predicted to be between 241 and $265 \mathrm{mmol} / \mathrm{kg}$. The sulfate group density is linearly dependent on acid concentration and hydrolysis temperature and not dependent on hydrolysis time. Maximum sulfate group density can only be achieved at the expense of yield. The results presented here provide a foundation for subsequent, sequential optimization using narrower parameter ranges, allowing further optimization of the hydrolysis conditions and potentially enabling higher yield.
\end{abstract}


Keywords: Cellulose nanocrystals, Acid hydrolysis, Central composite design, Yield, Sulfate group density

\section{Introduction}

Cellulose is a natural, non-toxic, biodegradable, high-molecular-weight polymer and one of the most abundant renewable materials, produced in massive quantities by nature each year. With the growing need for natural renewable materials, cellulose and cellulose derivatives are regaining much attention for high-end applications in recent years. Furthermore, the increased level of understanding and advances in the development of nanotechnologies create a need for nanoscale cellulose materials. Derived from long cellulose fibers, nanoscale cellulose particles are drawing growing interest from both industry and academia. Different terms have been used to refer to cellulose nanoparticles, including cellulose nanocrystals (CNCs), cellulose whiskers, cellulose nanowhiskers, and nanocrystalline cellulose. Here, the term CNCs is used to refer to crystalline cellulose particles with dimensions smaller than $1 \mu \mathrm{m}$.

Nanocellulosic materials are of great interest for a breadth of applications, ranging from reinforcing composite fillers to next generation biomedical and electronics applications (Shatkin et al., 2014). However, their widespread implementation is limited by their production cost, which is currently higher than the target of $\$ 2-5$ per pound for CNCs from virgin wood pulp (Cowie et al., 2014). While current costs may be suitable for niche applications, larger commercial markets cannot bear such high cost. To achieve broad market integration, the production cost must be substantially reduced to costs competitive with comparable nanoparticle additives. Successful realization of high volume CNC use for commercial markets therefore requires reduction of production costs, which may be partially achieved through substantial increase of CNC yield during the synthesis and production process.

For the production of CNCs, the classic and most widely used method is acid hydrolysis of a purified cellulose starting material. Different inorganic acids, most commonly sulfuric and 
hydrochloric acid, have been used for this purpose (Nickerson and Habrle, 1947; Rånby, 1951; Mukherjee and Woods, 1953; Battista et al., 1956; Revol et al., 1992; Araki et al., 1998). When the colloidal stability of CNC suspensions is important, sulfuric acid is preferable because, in addition to cleaving the cellulose microfibrils, it esterifies some of their surface hydroxyl groups (Marchessault et al., 1961). The obtained CNCs will then bear negative charges from dissociated surface sulfate groups $\left(\mathrm{p} K_{\mathrm{a}}=2.46\right.$, Wang et al., 2011). The resulting repulsive electrostatic forces between the negatively charged CNCs will prevent CNC aggregation. Thus, the surface charge density of CNCs plays a key role in the colloidal stability of their suspensions.

Besides the type of acid, many other experimental parameters affect the properties of $\mathrm{CNCs}$, including the starting material (e.g. wood pulp, cotton fibers, microcrystalline cellulose (MCC), bacterial cellulose), the acid concentration, the acid-to-cellulose ratio, the hydrolysis temperature, the hydrolysis time, the mode and speed of agitation, and the work-up procedure. The production of CNCs of a desired size or surface charge requires a detailed understanding of these effects. The choice of process conditions, however, will also inevitably affect CNC yield. Historically, hydrolysis conditions were optimized to produce CNCs that readily form colloidal liquid crystalline phases (Revol et al., 1992; Revol et al., 1994; Dong et al., 1998). Unfortunately, the high sulfuric acid concentration of 64-65 wt.\%, required to achieve good colloidal properties, results in low CNC yields on the order of 21-44\% (Dong et al., 1998; Hamad and Hu, 2010; Wang et al., 2012). Low yields contribute substantially to CNC production costs and maximizing CNC yield may generate significant cost reduction, assuming similar production processes.

The most efficient way to optimize a production process and maximize yield is the design of experiments (DOE) method. DOE is a systematic approach for determining the relationship between different process parameters $\left(x_{1}, x_{2}, x_{3}, \ldots\right)$ and process outputs $\left(y_{1}, y_{2}, y_{3}, \ldots\right)$. DOE approaches generally take less time and require fewer runs than traditional optimization approaches and enable the identification of parameter interactions and prediction of optimum process conditions for a desired output. Bondeson et al. (2006) were the first to use a DOE 
approach to study the production of CNCs from MCC by sulfuric acid hydrolysis. They applied response surface methodology (RSM) in combination with a Fractional Factorial Resolution V experimental design to optimize the concentration of MCC, sulfuric acid concentration, duration and temperature of hydrolysis, and duration of sonication. The obtained model predicted a maximum yield of $40 \%$ for an MCC concentration of $10.2 \mathrm{~g} / 100 \mathrm{~mL}$, an acid concentration of $63.5 \mathrm{wt} \%$, a hydrolysis temperature of $44{ }^{\circ} \mathrm{C}$, a hydrolysis time of $130 \mathrm{~min}$, and a sonication time of 30 min. Experimentally, however, the optimized conditions resulted in a CNC yield of only $30 \%$. More recently, Zou et al. (2012) used a three-factor, three-level orthogonal design to study the same process and reported experimental yields in excess of $82 \%$ and optimum reaction conditions of $33 \mathrm{wt} . \%$ for the acid concentration, $43{ }^{\circ} \mathrm{C}$ for the hydrolysis temperature, and 108 min for the hydrolysis time. Fan and $\mathrm{Li}$ (2012) used the same approach to determine the optimum reaction conditions for the preparation of $\mathrm{CNCs}$ from cotton pulp fibers by sulfuric acid hydrolysis. They reported a maximum experimental yield of $64 \%$ at an acid concentration of 64 wt.\%, a hydrolysis temperature of $50{ }^{\circ} \mathrm{C}$, and a hydrolysis time of $5 \mathrm{~h}$. Tang et al. (2011) used a Box-Behnken experimental design to optimize the preparation of $\mathrm{CNCs}$ from MCC with cation exchange resin in an ultrasonic reactor. The predicted optimum reaction conditions, i.e., a resinto-MCC ratio of $10(\mathrm{w} / \mathrm{w})$, a hydrolysis temperature of $48{ }^{\circ} \mathrm{C}$, and a hydrolysis time of $189 \mathrm{~min}$, resulted in an experimental yield of 50\%. Lu et al. (2013) used the same approach to optimize the preparation of $\mathrm{CNCs}$ from filter paper by ultrasonic wave and microwave-assisted sulfuric acid hydrolysis. They reported an experimental yield of $86 \%$ at optimum reaction conditions, i.e., a hydrolysis temperature of $70{ }^{\circ} \mathrm{C}$, a sulfuric acid concentration of $53 \%$, and a hydrolysis time of 1.5 h. Wang et al. (2012) used a Box-Wilson central composite design (CCD) to optimize the hydrolysis of bleached kraft eucalyptus pulp for minimal cellulose loss. The obtained model predicted a maximum $\mathrm{CNC}$ yield of $64 \%$ at a sulfuric acid concentration of $60 \mathrm{wt} \%$ and a hydrolysis temperature of $58{ }^{\circ} \mathrm{C}$. The influence of hydrolysis time on $\mathrm{CNC}$ yield was not discussed in detail and the highest experimental yield reported was $60 \%$. More recently, the team reported experimental yields in excess of $70 \%$ at acid concentrations of 58 and 62 wt.\% and 
hydrolysis temperatures of 56 and $50^{\circ} \mathrm{C}$, respectively (Wang et al., 2014; Chen et al., 2015). The experimental CNC yields were in good agreement with yields predicted by a kinetic model, which revealed a complex dependency of $\mathrm{CNC}$ yield on acid concentration, hydrolysis temperature, and hydrolysis time.

In this study, we use a three-factor, rotatable CCD to analyze the production of CNCs from softwood sulfite pulp by sulfuric acid hydrolysis and, specifically, to elucidate the relationships between the sulfuric acid concentration, hydrolysis temperature, hydrolysis time and CNC yield. The CCD has been developed in the vein of RSM, building on initial screening results from related studies to establish a design space and integrating subsequent experimentation for process optimization (Vining, 2011). In light of the sequential nature of RSM, this initial study encompasses a broad design space and its objectives are to 1) identify significant factors and factor interactions and 2) delineate a narrowed design space for subsequent experimentation. Because of its technological importance, the sulfate group density is included in this study as a second response variable.

\section{Materials and Methods}

\subsection{Materials}

Cellulose, in the form of dissolving-grade softwood sulfite pulp (Temalfa 93), was provided by Tembec, Inc. Sulfuric acid (96.4\%, certified ACS plus), sodium chloride (ACS certified), and sodium hydroxide $(0.02 \mathrm{~N}$, certified) were purchased from Fisher Scientific. The water used in the experiments was deionized (DI) water from a Millipore Direct-Q 5 ultrapure water system with $18.2 \mathrm{M} \Omega \cdot \mathrm{cm}$ resistivity at $25^{\circ} \mathrm{C}$.

\subsection{Methods}

\subsubsection{Experimental design and data analysis}

A three-factor, rotatable CCD with six center runs was generated with JMP statistical data analysis software (SAS Institute Inc., v8.0x). In this experimental design, the factor $a$, defining 
the radius of the circumscribed sphere, is 1.682 . The levels for each factor $(-a,-1,0,1, a)$ are listed in Table 1 and the design matrix is shown in Table 2. Factor levels were selected on the basis of literature values used for CNC production (Revol et al., 1992; Revol et al., 1994; Revol et al., 1998; Wang et al., 2012). Measured responses (output variables) are the CNC yield and the sulfate group density. Experiments were conducted in randomized order, generated by the software.

Table 1. Factors and design levels for the CCD

\begin{tabular}{lcccccc}
\hline & & \multicolumn{5}{c}{ Level } \\
\cline { 3 - 7 } Factor & Code & $-a$ & -1 & 0 & 1 & $a$ \\
\hline Sulfuric acid concentration (wt.\%) & $x_{1}$ & 51.6 & 55 & 60 & 65 & 68.4 \\
Hydrolysis temperature $\left({ }^{\circ} \mathrm{C}\right)$ & $x_{2}$ & 38.2 & 45 & 55 & 65 & 71.8 \\
Hydrolysis time (min) & $x_{3}$ & -21.1 & 30 & 105 & 180 & 231.1 \\
\hline
\end{tabular}


Table 2. Design matrix for the CCD

\begin{tabular}{lrrr}
\hline Run number & $x_{1}$ & $x_{2}$ & $x_{3}$ \\
\hline 1 & $-a$ & 0 & 0 \\
2 & -1 & -1 & 1 \\
3 & -1 & -1 & -1 \\
4 & -1 & 1 & 1 \\
5 & -1 & 1 & -1 \\
6 & 0 & 0 & 0 \\
7 & 0 & 0 & $-a$ \\
8 & 0 & 0 & 0 \\
9 & 0 & 0 & $a$ \\
10 & 0 & 0 & 0 \\
11 & 0 & 0 & 0 \\
12 & 0 & 0 & 0 \\
13 & 0 & $a$ & 0 \\
14 & 0 & $-a$ & 0 \\
15 & 0 & 0 & 0 \\
16 & 1 & -1 & 1 \\
17 & 1 & -1 & -1 \\
18 & 1 & 1 & -1 \\
19 & 1 & 1 & 1 \\
20 & $a$ & 0 & 0 \\
\hline
\end{tabular}

It is important to note in Table 1 that the $-a$ level of the hydrolysis time $\left(x_{3}\right)$, called for in Run 7 (Table 2), corresponds to a negative hydrolysis time that is experimentally not feasible. The goal of this CCD experiment is to minimize variable limitations and encompass the largest possible design space, enabling further optimization through subsequent experimentation around a smaller, refined design space. To this end, the hydrolysis times of 30 and 180 min were maintained as the -1 and 1 levels respectively, and only one run is impacted by the infeasible $-a$ level. Model analysis in subsequent sections accounts for the discrepancy.

Response values were analyzed using the least squares method to fit the data to equation 1 .

$$
y={ }_{0}+{ }_{i=1}^{n}{ }_{i} x_{i}+{ }_{i=1}^{n}{ }_{i i} x_{i}^{2}+{ }_{i=1 j=i+1}^{n 1}{ }_{i j} x_{i} x_{j}+
$$


In equation $1, y$ represents the predicted response, $x_{1}, x_{2}, \ldots x_{\mathrm{n}}$ are the coded values of the experimental factors, $\beta_{0}$ is the intercept, $\beta_{1}, \ldots \beta_{\mathrm{n}}$ are the linear coefficients, $\beta_{11}, \beta_{22}, \ldots \beta_{\mathrm{nn}}$ are the quadratic coefficients, $\beta_{12}, \beta_{23}, \ldots \beta_{\mathrm{n}-1 \mathrm{n}}$ are the interaction coefficients, and $\varepsilon$ is a random error with mean 0 and variance $\sigma^{2}$. The reported margins of error are the half-widths of the $95 \%$ confidence intervals.

\subsubsection{Cellulose hydrolysis}

Pulp sheets were milled in a Wiley Mini Mill (3383-L10, Thomas Scientific) to pass a 60mesh screen. Sulfuric acid was diluted to the desired concentration using ultrapure DI water. During this process, acid concentration was monitored with a hydrometer (Fisherbrand, 1.4801.550, Fisher Scientific). For the hydrolysis, $50 \mathrm{~mL}$ of sulfuric acid was first equilibrated to the desired hydrolysis temperature in a $250 \mathrm{~mL}$ three-neck round-bottom flask immersed in a recirculating water bath (Precision, circulating water bath, $0.1^{\circ} \mathrm{C}$ precision). $5 \mathrm{~g}$ of milled pulp was then added to the warm acid bath. The reaction mixture was stirred at $300 \mathrm{rpm}$ with a mechanical stirrer. The hydrolysis temperature was verified with a thermometer inside the flask.

At the desired hydrolysis time, the flask was removed from the water bath and its contents were poured into cold $\left(\sim 4^{\circ} \mathrm{C}\right)$ DI water for 10 -fold dilution and quenching of the reaction. The flask was rinsed with a small amount of DI water. Next, the suspension $(\sim 500 \mathrm{~mL})$ was centrifuged at $4550 \mathrm{~g}$ for $10 \mathrm{~min}$ (Thermo Electron Corporation, ICE Centra GP8R Refrigerated Centrifuge), and the supernatant was discarded. The sediment was redispersed in DI water and centrifuged again. The supernatant was again discarded and the sediment redispersed in DI water. The resulting gel was transferred to Spectra/Por 4 dialysis tubing with a molecular weight cut off of $12-14 \mathrm{kDa}$ and dialyzed against DI water until the $\mathrm{pH}$ of the water was neutral according to $\mathrm{pH}$ test strips (Micro Essential pHydrion). The resulting suspensions were then sonicated for 10 minutes at 40\% output with a Sonics VC 505 ultrasonic processor (500 W, 20 $\mathrm{kHz}$, Sonics \& Materials Inc) and then centrifuged again at $4550 \mathrm{~g}$ for $10 \mathrm{~min}$ for the removal of large particles. The analyzed samples were the supernatants after centrifugation. 


\subsubsection{Yield determination}

The yield of CNCs was calculated according to equation 2

$$
Y=\frac{w_{s} \times c_{s}}{w_{p}} \times 100 \%
$$

where $w_{\mathrm{s}}$, is the weight and $c_{\mathrm{s}}$ is the weight concentration of the filtered CNC suspension, and $w_{\mathrm{p}}$ is the weight of pulp used. $c_{\mathrm{s}}$ was determined by weighing aliquots of the filtered $\mathrm{CNC}$ suspension in aluminum sample pans before and after evaporation of the water in an oven at 80 ${ }^{\circ} \mathrm{C}$. The suspension weight concentration was calculated by dividing the net dry sample weight by the wet suspension weight (equation 3) where $w_{\mathrm{r}}$ is the weight of the sample pan with residue, $w_{0}$ is the weight of the empty sample pan, and $w_{\mathrm{a}}$ is the weight of the sample pan with the suspension.

$$
c_{s}=\frac{w_{r} \quad w_{0}}{w_{a} \quad w_{0}} \times 100 \%
$$

\subsubsection{Sulfate group density measurements}

CNCs prepared by sulfuric acid hydrolysis have sulfate groups on their surface resulting from partial esterification of the surface hydroxyl groups by the acid. The sulfate group density of the CNCs at the various hydrolysis conditions was determined by conductometric titration with a Mettler Toledo S47 SevenMulti pH/conductivity meter with an InLab 70 conductivity probe. Fifty grams of a 0.5 wt.\% CNC suspension, obtained by dilution of the original suspension with DI water, was weighed into a three-neck flat-bottom flask with a magnetic stir bar. The titration was carried out under nitrogen by drop-wise addition of $0.005 \mathrm{~N}$ aqueous $\mathrm{NaOH}$ from a bottle-top burette. Suspension conductivity as a function of $\mathrm{NaOH}$ addition volume was recorded and plotted. V-shaped titration curves were obtained and analyzed using 
two linear regressions of each range of the conductivity plot (Fig. 1). Sulfate group density, $\sigma_{\text {sulfate, }}$ was calculated according to equation 4

$$
\text { sulfate }=\frac{v_{\mathrm{NaOH}} \times c_{\mathrm{NaOH}}}{m_{\mathrm{CNC}}}
$$

where $v_{\mathrm{NaOH}}$ is the $\mathrm{NaOH}$ volume at the intersection of the two regressions, $c_{\mathrm{NaOH}}$ is the molar concentration of the $\mathrm{NaOH}$ solution, and $m_{\mathrm{CNC}}$ is the total mass of CNCs present in the flask.

Fig. 1. Representative conductometric titration curve (Run 1). The dashed line indicates the intersection of the linear regression lines from the two "arms" of the V-shaped titration curve.

\section{Results and Discussion}

The measured response values for yield and sulfate group density are listed in Table 3. 
Table 3. Measured CCD response values

\begin{tabular}{lccccc}
\hline Run number & $x_{1}($ wt. $\%)$ & $x_{2}\left({ }^{\circ} \mathrm{C}\right)$ & $x_{3}(\min )$ & Yield $(\%)$ & Sulfate group density $(\mathrm{mmol} / \mathrm{kg})$ \\
\hline 1 & 51.6 & 55 & 105 & 22.8 & 143 \\
2 & 55 & 45 & 180 & 24.8 & 161 \\
3 & 55 & 45 & 30 & 0.4 & - \\
4 & 55 & 65 & 180 & 54.8 & 190 \\
5 & 55 & 65 & 30 & 48.1 & 179 \\
6 & 60 & 55 & 105 & 55.1 & 279 \\
7 & 60 & 55 & -21.1 & - & - \\
8 & 60 & 55 & 105 & 55.7 & 271 \\
9 & 60 & 55 & 231.1 & 53.4 & 277 \\
10 & 60 & 55 & 105 & 54.5 & 293 \\
11 & 60 & 55 & 105 & 56.1 & 316 \\
12 & 60 & 55 & 105 & 59.5 & 339 \\
13 & 60 & 71.8 & 105 & 55.2 & 252 \\
14 & 60 & 38.2 & 105 & 17.3 & 279 \\
15 & 60 & 55 & 105 & 59.0 & 341 \\
16 & 65 & 45 & 180 & 6.7 & 328 \\
17 & 65 & 45 & 30 & 17.2 & 391 \\
18 & 65 & 65 & 30 & 15.7 & - \\
19 & 65 & 65 & 180 & 16.3 & - \\
20 & 68.4 & 55 & 105 & - & \\
\hline
\end{tabular}

As observed in Table 3, some response data are not available. The reaction conditions of 55 wt.\% $\left(x_{1}\right), 45^{\circ} \mathrm{C}\left(x_{2}\right)$, and 30 min $\left(x_{3}\right)$ were likely too mild to produce CNCs in Run 3 , resulting in insufficient $\mathrm{CNC}$ quantity for determination of the sulfate group density by conductometric titration. Run 7 required a negative hydrolysis time per the outer design points specified by the rotatable CCD design, and as a result was the only run that could not be completed. Run 20 lies at the edge of the CCD design sphere and required a sulfuric acid concentration of 68.4 wt.\%. Combined with a hydrolysis temperature of $55^{\circ} \mathrm{C}$ and hydrolysis time of $105 \mathrm{~min}$, this high acid concentration resulted in a dark brown suspension indicating a high degree of cellulose degradation and the presences of contaminants that cannot be selectively removed. As a result, isolation and purification of CNCs from Run 20 was not possible. Similarly, Run 19, representing the $(1,1,1)$ corner point of the experimental design, implemented harsh reaction 
conditions of $65 \mathrm{wt} . \%\left(x_{1}\right), 65{ }^{\circ} \mathrm{C}\left(x_{2}\right)$, and $180 \mathrm{~min}\left(x_{3}\right)$. While a yield is reported, this data point is suspect because sufficient sample was not available for conductometric titration to determine sulfate group density. A dark brown color was also noticed with this sample, suggesting sample contamination with degradation products that may have resulted in an inaccurate yield measurement.

\subsection{Yield model}

Yield data was analyzed in two ways for model development, first by assuming a yield of $0 \%$ for Runs 7 and 20 to develop a model that is based on the full 20-run experimental design, and second by excluding Runs 7 and 20 from the model, resulting in a CCD model that is missing the axial points $(0,0,-a)$ and $(a, 0,0)$. With respect to the 20 -run model, assuming yield values of $0 \%$ for Runs 7 and 20 makes practical physical sense. For Run 7, which requires a negative hydrolysis time, the yield must be $0 \%$ because a greater yield can only be obtained after a hydrolysis time greater than 0 min. For Run 20, which combines the highest sulfuric acid concentration (68.4 wt.\%) with a hydrolysis temperature of $55^{\circ} \mathrm{C}$ and a hydrolysis time of 105 min, the result is nearly complete degradation of the starting material, causing a very low, not practically measurable CNC yield.

Addressing the validity of excluding Runs 7 and 20 from the model, Giovannitti-Jensen and Myers have shown that, within a radius of 0.7 of the design space, a CCD with missing axial points $(-a, 0,0)$ and $(0,0, a)$ has similar result prediction quality as without exclusion of these points (Giovannitti-Jensen and Myers, 1989). Beyond the radius of 0.7, a CCD with missing axial points $(-a, 0,0)$ and $(0,0, a)$ has considerable instability of prediction variance around the spherical average prediction variance. The same should apply to a CCD with missing axial points $(0,0,-a)$ and $(a, 0,0)$. Thus, the option of excluding Runs 7 and 20 from the model will limit the model's region of reliable prediction to hydrolysis conditions within the ranges 56.5-63.5 wt.\% $\left(x_{1}\right), 48.0-62.0^{\circ} \mathrm{C}\left(x_{2}\right)$, and 52.5-157.5 $\mathrm{min}\left(x_{3}\right)$. Per Giovannitti-Jensen and Myers (1989), the fact that Runs 2-5, 9, and 16-19 lie outside this region has no bearing on the result prediction 
quality of the model. Excluding these axial points, represented by Runs 7 and 20, does result in loss of information about $x_{3}{ }^{2}$, but this issue may be addressed in subsequent experimentation as necessary and as intended by the RSM approach.

The obtained second-degree polynomial equations for the 20-run and 18-run models are provided in equations 5 and 6 , respectively.

$$
\begin{aligned}
& y=56.65 \quad 8.09 \times x_{1}+10.95 \times x_{2}+8.13 \times x_{3} \\
& 8.70 \times x_{1} \times x_{2} \quad 5.12 \times x_{1} \times x_{3} \quad 0.83 \times x_{2} \times x_{3} \\
& 15.97 \times x_{1}^{2} \quad 7.18 \times x_{2}^{2} \quad 10.56 \times x_{3}^{2} \\
& y=56.77 \quad 9.92 \times x_{1}+10.95 \times x_{2}+3.55 \times x_{3} \\
& 8.70 \times x_{1} \times x_{2} \quad 5.12 \times x_{1} \times x_{3} \quad 0.83 \times x_{2} \times x_{3} \\
& 19.42 \times x_{1}^{2} \quad 8.01 \times x_{2}^{2} \quad 4.82 \times x_{3}^{2}
\end{aligned}
$$

\subsubsection{Yield Model Analysis}

Table 4 lists selected results from the analysis of variance (ANOVA) and lack-of-fit test of the two regression models. The $p$-values for the ANOVA F-tests are below the significance level ( $\alpha=0.05$ ) for both models, which signifies that both models contain at least one significant regression parameter. The coefficients of determination $\left(\mathrm{R}^{2}\right)$ are 0.93 for the 20-run model and 0.97 for the 18-run model, indicating that the models account for 93 and $97 \%$ of the yield variability, respectively. However, the low $p$-values for the lack-of-fit test $(p<0.05)$ mean that the lack of fit is significant for both models at the $5 \%$ significance level, indicating that the second-degree polynomials do not adequately fit the data. The lack-of-fit test involves partitioning of the standard deviation of the residuals, which are the differences between the actual (observed) and predicted response values, into a random (pure) error that is independent of the model and a lack-of-fit error that depends on the functional part of the model and a comparison of the two. In this test, the significance level $\alpha$ gives the probability of inadvertently 
rejecting a model that has a larger lack-of-fit error than random error but is nevertheless adequate.

Table 4. Selected results from the analysis of variance (ANOVA) and lack-of-fit test and model validation statistics of the two second-degree regression models

\begin{tabular}{|c|c|c|}
\hline & 20-run model & 18-run model \\
\hline$p$-value (ANOVA F-test, $\alpha=0.05$ ) & 0.0001 & $<0.0001$ \\
\hline $\mathrm{R}^{2}$ & 0.93 & 0.97 \\
\hline Adjusted $\mathrm{R}^{2}$ & 0.86 & 0.94 \\
\hline$p$-value (lack-of-fit test, $\alpha=0.05$ ) & 0.0008 & 0.0074 \\
\hline $\mathrm{AIC}^{\mathrm{a}}$ & 183 & 161 \\
\hline $\mathrm{BIC}^{\mathrm{b}}$ & 161 & 127 \\
\hline $\mathrm{PRESS}^{\mathrm{c}}$ & 5518 & 3475 \\
\hline Predicted $\mathrm{R}^{2}$ & 0.46 & 0.55 \\
\hline
\end{tabular}

${ }^{a}$ Akaike information criterion

${ }^{\mathrm{b}}$ Bayesian information criterion

${ }^{c}$ predicted residual sum of squares

Both models have two statistically insignificant terms, $x_{1} \cdot x_{3}$ and $x_{2} \cdot x_{3}$ for the 20-run model and $x_{3}$ and $x_{2} \cdot x_{3}$ for the 18-run model, as illustrated in Table 5. 
Table 5. $t$-Ratios and $p$-values for the parameters of the two second-degree regression models

\begin{tabular}{lccccc}
\hline & \multicolumn{2}{c}{20 -run model } & & \multicolumn{2}{c}{ 18-run model } \\
\cline { 2 - 3 } \cline { 5 - 6 } Term & $t$ Ratio & Prob $>|t|$ & & $t$ Ratio & Prob $>|t|$ \\
\hline Intercept & 16.34 & $<.0001$ & & 27.58 & $<.0001$ \\
$x_{1}$ & -3.52 & 0.0056 & & -5.81 & 0.0004 \\
$x_{2}$ & 4.76 & 0.0008 & & 8.02 & $<.0001$ \\
$x_{3}$ & 3.53 & 0.0054 & & 2.08 & 0.0710 \\
$x_{1} \cdot x_{2}$ & -2.90 & 0.0160 & & -4.88 & 0.0012 \\
$x_{1} \cdot x_{3}$ & -1.70 & 0.1191 & & -2.87 & 0.0208 \\
$x_{2} \cdot x_{3}$ & -0.28 & 0.7887 & & -0.46 & 0.6551 \\
$x_{12}$ & -7.13 & $<.0001$ & & -10.54 & $<.0001$ \\
$x_{22}$ & -3.21 & 0.0094 & & -5.80 & 0.0004 \\
$x_{32}$ & -4.71 & 0.0008 & & -2.62 & 0.0309 \\
\hline
\end{tabular}

Model enhancement by backward elimination of insignificant terms, with the remaining terms staying unchanged, produces superior models in terms of predictive ability (lower PRESS values) that, however, still have $p$-values for the lack-of-fit test below the significance level ( $\alpha=$ 0.05) (Table 6). Elimination of the $x_{3}$ term from the 18-run model (equation 6) is not advisable because $x_{3}$ is contained in the $x_{1} \cdot x_{3}$ and $x_{3}^{2}$ terms, which are significant (Rawlings et al. 1998). Elimination of only the $x_{1} \cdot x_{3}$ term from the 20-run model (equation 5) did not significantly enhance the model (Table 6). Therefore, this option will be disregarded in the following discussion. 
Table 6. Selected results from the analysis of variance (ANOVA) and lack-of-fit test and model validation statistics of the two second-degree regression models with the insignificant terms eliminated

\begin{tabular}{|c|c|c|c|c|}
\hline & \multicolumn{3}{|c|}{ 20-run model ${ }^{\mathrm{a}}$ without } & \multirow{2}{*}{$\begin{array}{c}18 \text {-run model } \\
\text { without } x_{2} \cdot x_{3} \text { term }\end{array}$} \\
\hline & $x_{1} \cdot x_{3}$ term & $x_{2} \cdot x_{3}$ term & $x_{1} \cdot x_{3}$ and $x_{2} \cdot x_{3}$ terms & \\
\hline$\overline{p \text {-value (ANOVA F-test, } \alpha=0.05)}$ & 0.0001 & $<0.0001$ & $<0.0001$ & $<0.0001$ \\
\hline $\mathrm{R}^{2}$ & 0.91 & 0.93 & 0.91 & 0.97 \\
\hline Adjusted $\mathrm{R}^{2}$ & 0.84 & 0.88 & 0.85 & 0.95 \\
\hline$p$-value (lack-of-fit test, $\alpha=0.05$ ) & 0.0006 & 0.0012 & 0.0009 & 0.0115 \\
\hline $\mathrm{AIC}$ & 178 & 173 & 170 & 147 \\
\hline $\mathrm{BIC}$ & 164 & 159 & 161 & 124 \\
\hline PRESS & 5006 & 4020 & 3982 & 1811 \\
\hline Predicted $\mathrm{R}^{2}$ & 0.51 & 0.60 & 0.61 & 0.76 \\
\hline
\end{tabular}

a equation 5

b equation 6

To reduce the lack-of-fit error, at the risk of overfitting the model, we investigated the option of forward selection and introduced higher degree terms to the polynomial equation. In this study, addition of a single third-degree term, such as $x_{1}^{3}, x_{1}^{2} \cdot x_{2}$, or $x_{1} \cdot x_{2} \cdot x_{3}$, did not yield models with an insignificant lack of fit. However, addition of two third-degree terms resulted in three polynomials of similar significance and fit for both models (Table 7). Standard deviation of the data results in a range within which models that vary only by higher order terms provide a similar fit. The polynomial equations that predicted the highest maximum yields for the $20-$ and 18-run experiments are represented in equations 7 and 8, respectively.

$$
\begin{aligned}
& y=56.65 \quad 8.09 \times x_{1}+10.95 \times x_{2}+15.88 \times x_{3} \\
& 8.70 \times x_{1} \times x_{2} \quad 5.12 \times x_{1} \times x_{3} \quad 0.83 \times x_{2} \times x_{3} \\
& 15.97 \times x_{1}^{2} \quad 7.18 \times x_{2}^{2} \quad 10.56 \times x_{3}^{2} \\
& 13.22 \times x_{1}^{2} \times x_{3}+3.60 \times x_{1} \times x_{2} \times x_{3}
\end{aligned}
$$




$$
\begin{aligned}
& y=56.65 \quad 19.75 \times x_{1}+10.95 \times x_{2}+2.65 \times x_{3} \\
& 8.70 \times x_{1} \times x_{2} \quad 5.12 \times x_{1} x_{3} \quad 0.83 \times x_{2} \times x_{3} \\
& 23.71 \times x_{1}^{2} \quad 7.21 \times x_{2}^{2} \quad 2.73 \times x_{3}^{2} \\
& +10.72 \times x_{1} \times x_{3}^{2}+3.60 \times x_{1} \times x_{2} \times x_{3}
\end{aligned}
$$

Table 7 illustrates that addition of the third-degree terms (equations 7 and 8) results in statistically significant models with similar fit. Additionally, both models predict that only the $x_{2} \cdot x_{3}$ interaction parameter is not significant ( $p$-values of 0.6551 and 0.3237 for the 18 -run and 20-run models, respectively), suggesting continuity between the two models in terms of significance of interaction parameters.

Table 7. Selected results from the analysis of variance (ANOVA) and lack-of-fit test and model validation statistics of the two third-degree regression models

\begin{tabular}{lcc}
\hline & 20-run model $^{\mathrm{a}}$ & 18-run model $^{\mathrm{b}}$ \\
\hline$p$-value (ANOVA F-test, $\alpha=0.05)$ & $<0.0001$ & $<0.0001$ \\
$\mathrm{R}^{2}$ & 0.996 & 0.997 \\
Adjusted $\mathrm{R}^{2}$ & 0.991 & 0.992 \\
$p$-value (lack-of-fit test, $\alpha=0.05)$ & 0.3575 & 0.6551 \\
AIC & 157 & 172 \\
BIC & 109 & 93 \\
PRESS & 412 & 190 \\
Predicted $\mathrm{R}^{2}$ & 0.96 & 0.98 \\
\hline aequation 7 & & \\
bequation 8 & &
\end{tabular}

Fig. 2 graphically illustrates the improved third-degree model performance in representing actual measured yields. For the 20-run model, four data points lie outside the significance level curves with the second-degree model (Fig. 2a), but all data points lie within the significance level curves of the third-degree model (Fig. 2c). For the 18-run model, the data points are 
significantly closer to the predictive model with the third-degree model (Fig. 2d) than with the second-degree model (Fig. 2b).

Fig. 2. Plots of actual and predicted yield for (a) the second-degree 20-run model, (b) the seconddegree 18-run model, (c) the third-degree 20-run model, and (d) the third-degree 18-run model. In (b) and (d), the excluded runs are marked by an $\times$. The solid lines represent the model fit, the dashed lines are the significance level curves $(\alpha=0.05)$, and the dotted lines indicate the data means. The design center points are marked by open circles.

Another important criterion for the goodness of fit of regression models is the distribution of residuals around the mean, which allows estimation of unobservable experimental error. Fig. 3 illustrates plots of the yield residuals compared to predicted yield as well as the residual histograms, outlier box plots, and normal probability plots for both third-degree models. The residuals-by-predicted plots shows no systematic patterns for the residual distributions around the means that could indicate a problem. However, tests for the normality of the distributions indicate that the residuals of the third-degree 18-run model are not normally distributed, which is not the case for the second-degree models (equation 6 with and without $x_{2} \cdot x_{3}$ term). Addition of another third-degree term, specifically $x_{2}{ }^{3}, x_{1}{ }^{2} \cdot x_{2}$, or $x_{2} \cdot x_{3}{ }^{2}$, did not produce normally distributed residuals. The non-normal distribution of residuals might indicate that a third-degree polynomial is overfitting the 18-run yield model. Thus, for the 18-run case, the reduced second-degree model (equation 6 without the $x_{2} \cdot x_{3}$ term) might be the best model despite its significant lack of fit. A significant lack of fit can be a result of a small pure error, in which case the model will still provide accurate prediction.

In the 20-run case, however, the third-degree model (equation 7) is a better model, from a statistical point of view, than the reduced second-degree model (equation 5 without the $x_{2} \cdot x_{3}$ (and $\left.x_{1} \cdot x_{3}\right)$ term), because it results in a lower PRESS value (412 versus 4020 (3982)), an insignificant lack of fit, and a predicted $\mathrm{R}^{2}(0.96)$ within 0.2 of the adjusted $\mathrm{R}^{2}(0.99)$. In the enhancement of a 
regression model, one has to weigh the risk of an observed lack of fit being real against the risk of overfitting the model. Quoting George Box "All models are wrong, but some are useful”, we are of the opinion that, while equation 7 may be overfitting the model and the third-degree terms may have no physicochemical basis, analysis of this model may still provide valuable information.

Fig. 3. Plot of yield residual versus predicted yield $(a, c)$ and normal quantile plots $(b, d)$ for the 20-run (a, b) and 18-run (c, d) yield model. In a) and c), the dotted lines indicate the data means and the design center points are marked by open circles. In b) and d), the dashed lines are the Lilliefors confidence bounds and the upper scale is the normal quantile scale.

\subsubsection{Yield Model Factor and Interaction Effects}

Table 8 lists the $p$-values for each of the terms of the third-degree 20 -run yield model for a two-tailed $t$-test for the null hypothesis that the parameter is 0 .

Table 8. Parameter $p$-values for the third-degree 20-run yield model.

\begin{tabular}{ll}
\hline Term & $p$-value \\
\hline Intercept & $<.0001$ \\
$x_{1}$ & $<.0001$ \\
$x_{2}$ & $<.0001$ \\
$x_{3}$ & $<.0001$ \\
$x_{1} \cdot x_{2}$ & $<.0001$ \\
$x_{1} \cdot x_{3}$ & 0.0002 \\
$x_{2} \cdot x_{3}$ & 0.3237 \\
$x_{12}$ & $<.0001$ \\
$x_{22}$ & $<.0001$ \\
$x_{32}$ & $<.0001$ \\
$x_{12} \cdot x_{3}$ & $<.0001$ \\
$x_{1} \cdot x_{2} \cdot x_{3}$ & 0.0018 \\
\hline
\end{tabular}


As evident from Table 8 , all terms except the interaction term $x_{2} \cdot x_{3}$ are statistically significant $(p<0.05)$. In other words, the interaction between the hydrolysis temperature and the hydrolysis time does not have a statistically significant effect on CNC yield. The term $x_{2} \cdot x_{3}$ cannot be removed from the model, however, because the interaction is also contained in the term $x_{1} \cdot x_{2} \cdot x_{3}$, which is significant (Rawlings et al. 1998). Recall that the $x_{2} \cdot x_{3}$ interaction parameter is also insignificant in the second-degree yield models (Table 6). The finding that the $x_{2} \cdot x_{3}$ interaction does not significantly affect $\mathrm{CNC}$ yield is in agreement with the data reported by Wang et al. (2014), which showed strong effects of the acid concentration and hydrolysis temperature on CNC yield. The hydrolysis time affected CNC yield only during the initial period of the reaction, whereby the length of this period depended strongly on the other two factors. The present design space encompasses hydrolysis times as short as $30 \mathrm{~min}$, at which CNC yield still exhibited a dependency for all but the most drastic hydrolysis conditions (Wang et al., 2014). Therefore, the statistical significance of the $x_{3}$-term in the model is likely a result of the low -1 level for $x_{3}$.

The statistical significance or insignificance of interaction terms in a model is also evident in the factor interaction profiles of the model. Fig. 4 shows the factor interaction profiles for the third-degree 20-run yield model. The $x_{1} \cdot x_{2}$ and, if applicable, $x_{1} \cdot x_{3}$ interaction profiles for the reduced second-degree models are similar to those shown in Fig. 4. For interactions that are not statistically significant, the shapes of the two curves in each graph, representing the -1 and 1 conditions for the second factor, are nearly identical. Here, this is the case for the $x_{2} \cdot x_{3}$ interaction. However, in the third-degree 20-run yield model, the $x_{1} \cdot x_{2}$ and $x_{1} \cdot x_{3}$ interactions are statistically significant as evident from the different shapes of the two curves in the respective graphs.

Fig. 4. Factor interaction profiles for the third-degree 20-run yield model.

\subsubsection{Yield Optimization}


Fig. 5 illustrates the prediction profiles for the third-degree 20-run yield model for maximized yield. The prediction profiles for the reduced second-degree yield models are similar to those shown in Fig. 5.

Fig. 5. Yield prediction profiles for the third-degree 20-run yield model. The dashed lines indicate the $95 \%$ confidence intervals. The dotted lines mark the points of maximum yield.

The predicted maximum yields and optimum hydrolysis conditions for the four yield models are listed in Table 9. It should be noted that the optimum hydrolysis temperatures for the seconddegree 20-run models lie outside the design space, and the optimum temperature for the 18-run model lies outside the region of low prediction variance (0.7 radius). As a result, the maximum yield values predicted by these models have larger error margins than that predicted by the thirddegree 20-run model. It is anticipated that subsequent experimentation would verify a properly selected design space. The current models suggest an appropriate range of parameters for subsequent experimentation per RSM methodology. For instance, sulfuric acid concentrations above $62 \mathrm{wt} \%$ and hydrolysis times below $60 \mathrm{~min}$ are likely unnecessary and outside of the range of experimental interest for maximizing yield. 
Table 9. Maximum yield and optimum hydrolysis conditions for the reduced second-degree and the third-degree yield models.

\begin{tabular}{lcccc}
\hline & \multicolumn{3}{c}{ 20-run model $^{\mathrm{a}}$ without } \\
\cline { 2 - 5 } & $x_{2} \cdot x_{3}$ term & $\begin{array}{c}x_{1} \cdot x_{3} \text { and } x_{2} \cdot x_{3} \\
\text { terms }\end{array}$ & $\begin{array}{c}\text { 18-run model } \\
\text { without } x_{2} \cdot x_{3} \\
\text { term }\end{array}$ & $\begin{array}{c}\text { 20-run model } \\
\text { with } x_{1}{ }^{2} \cdot x_{3} \text { and } \\
x_{1} \cdot x_{2} \cdot x_{3} \text { terms }\end{array}$ \\
\hline Maximum yield (\%) & $67.9 \pm 10.3$ & $66.5 \pm 9.9$ & $66.2 \pm 5.7$ & $69.1 \pm 2.7$ \\
Sulfuric acid concentration (wt.\%) & 56.7 & 57.2 & 57.2 & 58.3 \\
Hydrolysis temperature $\left({ }^{\circ} \mathrm{C}\right)$ & 66.6 & 66.0 & 64.9 & 63.7 \\
Hydrolysis time (min) & 145.8 & 133.9 & 155.2 & 155.8 \\
\hline
\end{tabular}

a equation 5

b equation 6

c equation 7

The predicted maximum yields and optimum hydrolysis conditions are in good agreement with the findings of Wang et al. (Wang et al., 2012; Wang et al., 2014; Chen et al., 2015), who predicted a maximum CNC yield of around $70 \%$ at sulfuric acid concentrations between 58 and 62 wt.\%, hydrolysis temperatures between 50 and $60{ }^{\circ} \mathrm{C}$, and hydrolysis times between 30 and $180 \mathrm{~min}$.

Fig. 6 shows contour plots of the third-degree 20-run yield model for each of the binary combinations of the three factors with the third factor fixed at its 0 level. The plots are scaled from -1 to 1 , beyond which range the prediction quality of the $\mathrm{CDD}$ deteriorates rapidly (Giovannitti-Jensen and Myers, 1989). The contour plots of the reduced second-degree models are similar to those shown in Fig. 6. Circular contour lines occur when the interaction between the two factors is negligible whereas non-circular contour lines indicate significant factor interaction. The non-circular contour lines of Fig. $6 \mathrm{a}$ and $6 \mathrm{~b}$ illustrate significant interaction between the sulfuric acid concentration $\left(x_{1}\right)$ and the hydrolysis temperature $\left(x_{2}\right)$ as well as between the acid concentration and hydrolysis time $\left(x_{3}\right)$. The nearly circular contour lines of Fig. 6c suggest a lack of interaction between the hydrolysis temperature and hydrolysis time. 
Fig. 6. Contour plots for the third-degree 20-run yield model.

The corresponding response surface plots are illustrated in Fig. 7. The surfaces are drawn from -1 to 1 and the third factor is fixed at its 0 level. The shapes of the contour lines and response surfaces suggest that a region exists within the design space that may produce a maximum yield. However, as illustrated in Fig. 7a-7c, one corner of the response surface is near the point at which a yield maximum exists, and requires subsequent experimentation per RSM to more accurately define the local design space.

Fig. 7. Perspective plots for the third-degree 20 -run yield model. The black circles represent the actual yield values with the design center points marked by open circles.

\subsubsection{Verification of Yield Model Prediction}

Because of subsequent revision of the model, testing of the model occurred at conditions slightly different from the optimum conditions. Hydrolysis conditions implementing an acid concentration of $57 \mathrm{wt} . \%$, a hydrolysis temperature of $65{ }^{\circ} \mathrm{C}$, and a hydrolysis time of $125 \mathrm{~min}$ resulted in $63.6 \%$ yield, which is within the error margins of the yields predicted by the seconddegree models but outside those of the yield predicted by the third-degree model (Table 10). Subsequent analysis within a more narrow design space may yield a more accurate model and experimental yields within the error margins of model predictions. 
Table 10. Predicted yields at an acid concentration of $57 \mathrm{wt} \%$, a hydrolysis temperature of 65 ${ }^{\circ} \mathrm{C}$, and a hydrolysis time of $125 \mathrm{~min}$.

\begin{tabular}{|c|c|c|c|c|}
\hline & \multicolumn{2}{|c|}{ 20-run model ${ }^{\mathrm{a}}$ without } & \multirow{2}{*}{$\begin{array}{c}\text { 18-run model } \\
\text { without } x_{2} \cdot x_{3} \\
\text { term }\end{array}$} & \multirow{2}{*}{$\begin{array}{l}20 \text {-run model } \\
\text { with } x_{1}^{2} \cdot x_{3} \text { and } \\
x_{1} \cdot x_{2} \cdot x_{3} \text { terms }\end{array}$} \\
\hline & $x_{2} \cdot x_{3}$ term & $\begin{array}{c}x_{1} \cdot x_{3} \text { and } x_{2} \cdot x_{3} \\
\text { terms }\end{array}$ & & \\
\hline Predicted yield (\%) & $67.0 \pm 8.8$ & $66.2 \pm 9.4$ & $65.3 \pm 5.5$ & $67.0 \pm 2.6$ \\
\hline
\end{tabular}

${ }^{a}$ equation 5

b equation 6

${ }^{c}$ equation 7

\subsection{Sulfate Group Density Model}

As discussed in Section 3, sulfate group density data are not available for Runs 3 (-1, -1,1), $7(0,0,-a), 19(1,1,1)$, and $20(a, 0,0)$. The polynomial model developed from the regression of the sulfate group density measurements, under exclusion of Runs 3, 7, 19, and 20, is provided in equation 9 .

$$
\begin{aligned}
& y=296.17+95.88 \times x_{1}+18.76 \times x_{2}+3.88 \times x_{3} \\
& +24.20 \times x_{1} \times x_{2}+16.20 \times x_{1} \times x_{3}+15.70 \times x_{2} \times x_{3} \\
& +2.86 \times x_{1}^{2} \quad 6.25 \times x_{2}^{2} \quad 9.08 \times x_{3}^{2}
\end{aligned}
$$

\subsubsection{Sulfate Group Density Model Analysis}

The sulfate group density model has a high $\mathrm{R}^{2}$ value of 0.95 , a $p$-value for the ANOVA Ftest of 0.0026 and a $p$-value for the lack-of-fit test of 0.6910 at the $5 \%$ significance level. These $p$-values signify that the model has at least one significant regression parameter and that there is not enough evidence to reject the null hypothesis that the model is adequate.

Fig. 8 illustrates a plot of the sulfate group density residual compared to predicted sulfate group density as well as the residual histogram, outlier box plot, and normal probability plot for 
the model. The residuals-by-predicted plot showed no systematic pattern for the residual distribution around the mean that could indicate a problem. However, a test for normality indicates that the residuals of this model are not from a normally distributed population. Analysis of factor and interaction effects in the following section indicates that the residual distribution around the mean of this model becomes irrelevant.

Fig. 8. Plot of sulfate group density residual compared to predicted sulfate group density (a) and normal quantile plot (b) for the second-degree sulfate group density model. In a), the dotted line indicates the data mean and the design center points are marked by open circles. In b), the dashed lines are the Lilliefors confidence bounds and the upper scale is the normal quantile scale.

\subsubsection{Sulfate Group Density Model Factor and Interaction Effects}

Table 11 lists the $p$-values for each of the terms of the sulfate group density model for a two-tailed $t$-test for the null hypothesis that the parameter is 0 .

Table 11. Parameter $p$-values for the second-degree sulfate group density model.

\begin{tabular}{lc}
\hline Term & $p$-value \\
\hline Intercept & $<.0001$ \\
$x_{1}$ & 0.0001 \\
$x_{2}$ & 0.0544 \\
$x_{3}$ & 0.7429 \\
$x_{1} \cdot x_{2}$ & 0.2659 \\
$x_{1} \cdot x_{3}$ & 0.4430 \\
$x_{2} \cdot x_{3}$ & 0.4566 \\
$x_{12}$ & 0.8120 \\
$x_{22}$ & 0.4101 \\
$x_{32}$ & 0.4596 \\
\hline
\end{tabular}

As observed in Table 11, only the intercept and the acid concentration are statistically significant $(p<0.05)$. Elimination of the quadratic terms from the model results in a slightly 
superior model with increased adjusted $\mathrm{R}^{2}$ and decreased AIC and BIC values (Table 12). Further elimination of the parameter interaction terms causes a slight decrease in the adjusted $\mathrm{R}^{2}$ value but also an increase in the predicted $\mathrm{R}^{2}$ and decreases in the AIC and PRESS values. Additionally, removal of all second-degree terms $\left(\beta_{\mathrm{ii}} x_{\mathrm{i}}^{2}, \beta_{\mathrm{ij}} x_{\mathrm{i}} x_{\mathrm{j}}\right)$ from the model results in an increase of the $p$-value for the hydrolysis temperature $\left(x_{2}\right)$ above the $5 \%$ significance level (0.0430). The $p$-value for the hydrolysis time $\left(x_{3}\right)$, however, remains below at a value of 0.9779 .

Table 12. Selected results from the analysis of variance (ANOVA) and lack-of-fit test and model validation statistics for the stepwise enhancement of the sulfate group density model by backward elimination.

\begin{tabular}{lcccc} 
Eliminated terms & none & $\beta_{\mathrm{ii}} x_{\mathrm{i}}^{2}$ & $\beta_{\mathrm{ii}} x_{\mathrm{i}}^{2}$ and $\beta_{\mathrm{ij}} x_{\mathrm{i}} x_{\mathrm{j}}$ & $\beta_{\mathrm{ii}} x_{\mathrm{i}}^{2}, \beta_{\mathrm{ij}} x_{\mathrm{i}} x_{\mathrm{j}}$, and $\beta_{3} x_{3}$ \\
\hline$p$-value (ANOVA F-test, $\alpha=0.05)$ & 0.0026 & $<0.0001$ & $<0.0001$ & $<0.0001$ \\
$\mathrm{R}^{2}$ & 0.950 & 0.941 & 0.903 & 0.903 \\
Adjusted R & 0.880 & 0.902 & 0.879 & 0.888 \\
$p$-value (lack-of-fit test, $\alpha=0.05)$ & 0.6910 & 0.8453 & 0.6312 & 0.3335 \\
AIC & 220 & 172 & 159 & 154 \\
BIC & 162 & 157 & 157 & 155 \\
PRESS & 9528 & 12329 & 11565 & 9769 \\
Predicted R & 0.873 & 0.835 & 0.846 & 0.870 \\
\hline
\end{tabular}

The finding that the hydrolysis time does not significantly affect sulfate group density suggests that the esterification of surface hydroxyl groups during cellulose hydrolysis is rapid and reaches its final level within $30 \mathrm{~min}$, except possibly at the conditions $55 \mathrm{wt} . \%\left(x_{1}\right), 45^{\circ} \mathrm{C}$ $\left(x_{2}\right)$, and $30 \mathrm{~min}\left(x_{3}\right)$ for which data are not available. This finding is in agreement with data reported by Chen et al. (2015), which show that hydrolysis times in excess of 30 min have a limited effect on $\mathrm{CNC}$ sulfate content at acid concentrations of $62\left(x_{2} \geq 50^{\circ} \mathrm{C}\right)$ or 64 wt.\% $\left(x_{2}=45^{\circ} \mathrm{C}\right)$ or a hydrolysis temperature of $70{ }^{\circ} \mathrm{C}\left(x_{1}=56\right.$ wt. $\left.\%\right)$. CNC sulfur contents at a hydrolysis time of 30 min have been shown to be insignificant at an acid concentration of 56 wt.\% and a hydrolysis temperature of $50^{\circ} \mathrm{C}$ (Chen et al., 2015). This observation may indicate 
that the statistical insignificance of the $x_{3}$-term may be a consequence of the missing $(-1,-1,-1)$ data point.

Elimination of the $x_{3}$ and all second-degree terms from the model (equation 9) gives the reduced first-degree model represented by equation 10 .

$$
y=280.98+90.20 \times x_{1}+17.12 \times x_{2}
$$

This model has a slightly lower adjusted $\mathrm{R}^{2}$ value $(0.888)$ than the model obtained by elimination of only the quadratic terms (0.902) but has a higher predicted $\mathrm{R}^{2}$ value and lower AIC, BIC, and PRESS values (Table 12). Its adjusted $\mathrm{R}^{2}$ value is higher and its AIC and BIC values are lower than those of the original model (equation 9). The performances of the original second-degree and reduced first-degree models (equations 9 and 10) are compared in Fig. 9.

Fig. 9. Plot of actual versus predicted sulfate group density for a) the second-degree sulfate group density model and b) the reduced first-degree sulfate group density model. The solid line represents the line of fit, the dashed lines are the significance level curves $(\alpha=0.05)$, and the dotted line indicates the data mean. The design center points are marked by open circles.

Fig. 10 illustrates the plots from the residual analysis of the reduced first-degree sulfate group density model. A normality test signifies that the residual data is likely from a normal distribution. Thus, the reduced first-degree sulfate group density model (equation 10) satisfies all requirements for an adequate, valid regression model. It should be kept in mind, however, that this model is based on an incomplete data set. The linear relationship between the sulfate group density and the acid concentration, stipulated by the model, is in disagreement with the findings of Chen et al. (2015), who observed lower sulfur contents at an acid concentration of 64 wt.\% than at an acid concentration of $62 \mathrm{wt} . \%$. 
Fig. 10. Plot of sulfate group density residual versus predicted sulfate group density (a) and normal quantile plot (b) for the reduced first-degree sulfate group density model. The design center points are marked by open circles. In a), the dotted line indicates the data mean. In b), the dashed lines are the Lilliefors confidence bounds and the upper scale is the normal quantile scale.

\subsubsection{Sulfate Group Density Model Predictions}

The model predicts a minimum sulfate group density of $174 \pm 27 \mathrm{mmol} / \mathrm{kg}$ at the -1 levels of the acid concentration (55 wt.\%) and hydrolysis temperature $\left(45^{\circ} \mathrm{C}\right)$ and a maximum sulfate group density of $388 \pm 30 \mathrm{mmol} / \mathrm{kg}$ at the +1 levels of the acid concentration $(65 \mathrm{wt} . \%)$ and hydrolysis temperature $\left(65^{\circ} \mathrm{C}\right)$. While the data reported by Chen et al. (2015) suggest that the model predictions for $(-1,-1)$ and $(1,1)$ may be inaccurate, an ongoing study focused on subsequent experimentation based on the results presented here and that will be separately published, has revealed a preliminary model based on a complete data set predicting within 70 and $3 \%$, respectively ( $102 \pm 36$ and $401 \pm 36$, respectively), of the model presented here. For the hydrolysis conditions predicted by the yield models to give maximum yield, the current sulfate group density model predicts a sulfate group density between $241 \pm 22 \mathrm{mmol} / \mathrm{kg}\left(x_{1}=56.7 \mathrm{wt}\right.$.\%, $\left.x_{2}=66.6{ }^{\circ} \mathrm{C}\right)$ and $265 \pm 18 \mathrm{mmol} / \mathrm{kg}\left(x_{1}=58.3 \mathrm{wt} . \%, x_{2}=63.7^{\circ} \mathrm{C}\right)$.

\subsubsection{Verification of Sulfate Group Density Model Prediction}

Hydrolysis conditions implementing an acid concentration of $58 \mathrm{wt} \%$, a hydrolysis temperature of $59{ }^{\circ} \mathrm{C}$, and a hydrolysis time of $160 \mathrm{~min}$ resulted in a sulfate group density of 228 $\mathrm{mmol} / \mathrm{kg}$, which was $9 \%$ lower than the predicted sulfate group density of $252 \pm 15 \mathrm{mmol} / \mathrm{kg}$. As stated in Section 3.1.4, subsequent analysis within a more narrow design space may improve the accuracy of model predictions.

\section{Conclusions}


A rotatable central composite design was used to evaluate the impact of processing conditions on the yield and sulfate group density of CNCs produced using sulfuric acid hydrolysis of wood pulp. For sulfuric acid concentrations, hydrolysis temperatures, and hydrolysis times within the ranges $55-65 \mathrm{wt} . \%$, 45-65 ${ }^{\circ} \mathrm{C}$, and $30-180 \mathrm{~min}$, respectively, this study demonstrates that all three parameters significantly affect CNC yield and these effects are reasonably well described by second-degree polynomials in three variables missing up to two interaction terms or having two additional third-degree terms. The sulfate group density of CNCs, as measured by conductometric titration, is not significantly affected by the hydrolysis time. The effects of acid concentration and hydrolysis temperature on the sulfate group density of CNCs are adequately described by a first-degree polynomial in two variables.

The regression models may be used to predict yield and property values for specific hydrolysis conditions or determine a set of hydrolysis conditions for a desired yield or sulfate group density. The yield regression models predict a maximum yield of 66-69\% at optimum process conditions of $57-58 \mathrm{wt} . \%\left(x_{1}\right), 64-67^{\circ} \mathrm{C}\left(x_{2}\right)$, and $134-156 \mathrm{~min}\left(x_{3}\right)$. At these conditions, the sulfate group density is predicted to be between 241 and $265 \mathrm{mmol} / \mathrm{kg}$.

For this study, a large design space was chosen to avoid experimental limitations and maximize potential to determine interaction parameters over a broad range of processing conditions. The results from this work establish the design space for subsequent experimental design to narrow the design space and determine whether additional improvements in yield may be obtained.

\section{Acknowledgements}

This material is based upon work supported by the National Research Initiative of the USDA Cooperative State Research, Education and Extension Service under Grant 2005-3550416088 and the National Science Foundation under Grants CHE-0724126 and DMR-0907567. Moreover, this work was supported in part by the Virginia Agricultural Experiment Station and the USDA National Institute of Food and Agriculture, Hatch/Multi State project 1002269. Partial 
funding from the Institute for Critical Technology and Applied Science, the Macromolecules and Interfaces Institute, and Omnova Solutions, Inc., and donation of the wood pulp by Tembec, Inc., are also acknowledged.

\section{References}

Araki, J., Wada, M., Kuga, S., Okano, T., 1998. Flow properties of microcrystalline cellulose suspension prepared by acid treatment of native cellulose. Colloids Surf., A 142, 75-82.

Battista, O.A., Coppick, S., Howsmon, J.A., Morehead, F.F., Sisson, W.A. 1956. Level-Off Degree of Polymerization-Relation to polyphase structure of cellulose fibres. Ind. Eng. Chem. $48,333-335$.

Beck-Candanedo, S., Roman, M., Gray, D.G. 2005. Effect of reaction conditions on the properties and behavior of wood cellulose nanocrystal suspensions. Biomacromolecules 6, 10481054.

Bondeson, D., Mathew, A., Oksman, K. 2006. Optimization of the isolation of nanocrystals from microcrystalline cellulose by acid hydrolysis. Cellulose 13, 171-180.

Chen, L., Wang, Q., Hirth, K., Baez, C., Agarwal, U.P., Zhu, J.Y. 2015. Tailoring the yield and characteristics of wood cellulose nanocrystals (CNC) using concentrated acid hydrolysis. Cellulose 22, 1753-1762.

Cowie, J., Bilek, E.M., Wegner, T.H., Shatkin, J. 2014. Market projections of cellulose nanomaterial-enabled products - Part 2: Volume estimates. TAPPI J. 13, 57-69. 
Dong, X.M., Revol, J.F., Gray, D.G. 1998. Effect of microcrystalline preparation conditions on the formation of colloid crystals of cellulose. Cellulose 1998, 5, 19-32.

Fan, J.S., Li, Y.H. 2012. Maximizing the yield of nanocrystalline cellulose from cotton pulp fiber. Carbohydr. Polym. 88, 1184-1188.

Giovannitti-Jensen, A., Myers, R.H. 1989. Graphical assessment of the prediction capability of response surface designs. Technometrics 31, 159-171.

Hamad, W.Y., Hu, T.Q. 2010. Structure-process-yield interrelations in nanocrystalline cellulose extraction. Can. J. Chem. Eng. 88, 392-402.

Jiang, F., Esker, A. R., Roman, M. 2010. Acid-catalyzed and solvolytic desulfation of $\mathrm{H}_{2} \mathrm{SO}_{4}{ }^{-}$ hydrolyzed cellulose nanocrystals. Langmuir 26, 17919-17925.

Lu, Z.X., Fan, L.W., Zheng, H.Y., Lu, Q.L., Liao, Y.Q., Huang, B.A. 2013. Preparation, characterization and optimization of nanocellulose whiskers by simultaneously ultrasonic wave and microwave assisted. Bioresour. Technol. 46, 82-88.

Marchessault, R.H., Morehead, F.F., Koch, M.J., 1961. Some hydrodynamic properties of neutral suspensions of cellulose crystallites as related to size and shape. J. Colloid Sci. 16, 327-344.

Mukherjee, S.M., Woods, H.J., 1953. X-ray and electron microscope studies of the degradation of cellulose by sulphuric acid. Biochim. Biophys. Acta 10, 499-511.

Nickerson, R.F.; Habrle, J.A. 1947. Cellulose intercrystalline structure. Ind. Eng. Chem. 39, $1507-1512$. 
Rawlings, J.O.; Pantula, S.G.; Dickey, D.A. Applied Regression Analysis. A Research Tool. 2nd ed.; Springer: New York, 1998.

Rånby, B.G. 1951. III. Fibrous macromolecular systems. Cellulose and muscle. The colloidal properties of cellulose micelles. Discussions Faraday Soc. 11, 158-164.

Revol, J.-F., Bradford, H., Giasson, J., Marchessault, R.H., Gray, D.G. 1992. Helicoidal selfordering of cellulose microfibrils in aqueous suspension. Int. J. Biol. Macromol. 14, 170-172.

Revol, J.-F., Godbout, L., Dong, X.M., Gray, D.G., Chanzy, H., Maret, G. 1994. Chiral nematic suspensions of cellulose crystallites; phase separation and magnetic field orientation. Liq. Cryst. $16,127-134$.

Revol, J.-F., Godbout, L., Gray, D.G. 1998. Solid self-assembled films of cellulose with chiral nematic order and optically variable properties. J. Pulp Pap. Sci. 24, 146-149.

Shatkin, J., Wegner, T.H., Bilek, E.M., Cowie, C. 2014. Market projections of cellulose nanomaterial-enabled products - Part 1: Applications. TAPPI J. 13, 9-16.

Tang, L.-R., Huang, B., Ou, W., Chen, X.R., Chen, Y.D. 2011. Manufacture of cellulose nanocrystals by cation exchange resin-catalyzed hydrolysis of cellulose. Bioresour. Technol. 102, 10973-10977.

Vining, G. 2011. Technical advice: Design of experiments, response surface methodology, and sequential experimentation. Qual. Eng. 23, 217-220. 
Wang, H.Z., Qian, C., Roman M. 2011. Effects of pH and salt concentration on the formation and properties of chitosan-cellulose nanocrystal polyelectrolyte-macroion complexes. Biomacromolecules 12, 3708-3714.

Wang, Q.Q., Zhu, J.Y., Reiner, R.S., Verrill, S.P., Baxa, U., McNeil, S.E., 2012. Approaching zero cellulose loss in cellulose nanocrystal (CNC) production: recovery and characterization of cellulosic solid residues (CSR) and CNC. Cellulose 19, 2033-2047.

Wang, Q.Q., Zhao, X.B., Zhu, J.Y., 2014. Kinetics of strong acid hydrolysis of a bleached kraft pulp for producing cellulose nanocrystals (CNCs). Ind. Eng. Chem. Res. 53, 11007-11014.

Zou, P.P., Zhang, P., Gao, D., Xia, Q.N. 2012. Process' optimization of nanocrystalline cellulose and its properties. Appl. Mech. Mater. 200, 373-376. 